Präv Gesundheitsf 2019 · 14:218-222 https://doi.org/10.1007/s11553-018-0699-1 Eingegangen: 15. Oktober 2018

Online publiziert: 6. Februar 2019

(c) Der/die Autor(en) 2019

CrossMark
Angenommen: 19. Dezember 2018

Bianca Biallas (1D) - Lea Anna Lisa Dejonghe - Janika Meyer · Sina Humm · Ingo Froböse · Andrea Schaller

Institut für Bewegungstherapie und bewegungsorientierte Prävention und Rehabilitation, Deutsche Sporthochschule Köln, Köln, Deutschland

\section{Zusammenarbeit in der} betrieblichen Gesundheitsförderung - eine qualitative Analyse der Erwartungen von Arbeitgebern und gesetzlichen Krankenkassen

\section{Einleitung}

Technologischer Fortschritt, zunehmende Flexibilisierung und verstärkter Wettbewerbsdruck führen zu erhöhten Anforderungen am Arbeitsplatz. Aus diesem Grund nimmt die Bedeutung der betrieblichen Gesundheitsförderung (BGF) in der Arbeitswelt zu [13].

Bei der Umsetzung der BGF oder auch des betrieblichen Gesundheitsmanagements (BGM) ist eine Vielzahl von Akteuren beteiligt $[3,14]$. Neben den Arbeitnehmern und weiteren Experten z. B. aus den Bereichen Arbeitsschutz oder Arbeitssicherheit etc. sind Arbeitgeber und die gesetzlichen Krankenkassen (GKVen) wichtige Interessensvertreter [8]. Durch das „Gesetz zur Stärkung der Gesundheitsförderung und Prävention“ werden die Zuständigkeiten und Verantwortlichkeiten der gesetzlichen Unfallund Rentenversicherungsträger sowie eine trägerübergreifende Zusammenarbeit betont [10, 12]. Vor dem Hintergrund des Präventionsgesetzes mit den vielen beteiligten Akteuren und individuellen Zielsetzungen ergeben sich Herausforderungen bei der erfolgreichen Umsetzung von Präventionsmaßnahmen. Neben den vielfältigen Inhalten müssen auch die verschiedensten Ziele, Interessen und Handlungsmöglichkeiten bei der Implementierung und nachhaltigen Umsetzung von Maßnahmen der BGF Berücksichtigung finden [8].

Herausfordernd sind die teilweise abweichenden Erwartungen der unterschiedlichen Akteure in Bezug auf Präventionsmaßnahmen im betrieblichen Setting. Die Erwartungen von Arbeitnehmerseite liegen primär in der Verbesserung der eigenen Gesundheit [15]. Oftmals verbinden die Arbeitnehmer die Ziele der BGF aber vielmehr mit den Zielen der Arbeitgeber, wie der Reduktion von krankheitsbedingten Fehlzeiten sowie der Erhöhung der Leistungsfähigkeit [15]. Die Erwartungen von Arbeitgeberseite beinhalten u. a. eine bessere Gesundheit, eine höhere Leistungsfähigkeit, eine Steigerung der Arbeitsmotivation sowie eine Verbesserung der Arbeitsbedingungen und des Images [1]. Die Erwartungen der GKVen liegen laut Medizinischem Dienst des Spitzenverbandes Bund der Krankenkassen (MDS) im betrieblichen Kontext in der Verhütung von Muskel-Skelettsowie psychischer Erkrankungen. Zudem sollen die gesundheitsfördernden Potentiale in der Arbeitswelt gesteigert werden [9].

Die Erwartungen der Arbeitnehmer an die Ziele der Angebote sind bereits untersucht [2]. Die vorliegende Arbeit fokussiert sich auf die Erwartungen der Stakeholder Arbeitgeber und gesetzlichen Krankenversicherung hinsichtlich ihrer Zusammenarbeit vor dem Hintergrund des Präventionsgesetzes. Auf dieser Grundlage sollen die Rahmenbedingungen für die Umsetzung der Wünsche von Arbeitnehmern (AN) optimaler umgesetzt werden. Diese sind bei der organisatorischen Kooperation im ersten Schritt jedoch nicht involviert und werden daher im Rahmen dieser Publikation nicht berücksichtigt.

Es ergibt sich folgende Fragestellung für die vorliegende Arbeit:

- Welche Erwartungen haben die Arbeitgeber und die Vertreter der gesetzlichen Krankenversicherung (GKV) im Hinblick auf die Zusammenarbeit bei Maßnahmen der BGF?

\section{Methode}

Vor dem Hintergrund der Fragestellung wurde ein qualitatives Studiendesign gewählt, um einen vertiefenden Einblick in die Perspektiven der Stakeholder gewinnen zu können. Zwei weibliche, geschulte Personen der Autorengruppe führten da$\mathrm{zu}$ leitfadengestützte Interviews durch. Die Auswahl der Interviewpartner erfolgte auf Grundlage vorhandener Ko- 
Tab. 1 Zusammensetzung der Stichprobe in Bezug auf Alter, Geschlecht, Betriebszugehörigkeit und Betriebsgröße

\begin{tabular}{l|l|l|l} 
& Geschlecht & Alter & Betriebszugehörigkeit \\
& $\mathbf{m} / \mathbf{w}$ & Jahre & Jahre \\
\hline Arbeitgeber $(n=6)$ & $3 / 3$ & $37-60$ & $3-40$ \\
\hline Vertreter der GKV $(n=6)$ & $3 / 3$ & $31-62$ & $1,5-30$ \\
Gesamtgruppe & $6 / 6$ & $28-61$ & $1,5-40$ \\
\hline$m$ männlich, $w$ weiblich, GKV Gesetzliche Krankenversicherung & \\
\hline
\end{tabular}

Tab. 2 Hauptfragen für die Befragungsgruppe der Arbeitgeber und Vertreter der gesetzlichen Krankenversicherung

\begin{tabular}{|c|c|c|}
\hline Kategorie & Fragestellung Arbeitgeber & GKV \\
\hline \multirow[t]{2}{*}{$\begin{array}{l}\text { Präventionsmaß- } \\
\text { nahmen im Betrieb }\end{array}$} & \multirow{2}{*}{$\begin{array}{l}\text { (Nun aus Arbeitgebersicht): } \\
\text { In welcher Form erwarten Sie dabei } \\
\text { Unterstützung von den } \\
\text { Sozialversicherungsträgern? }\end{array}$} & $\begin{array}{l}\text { Was erwartet die GKV von den } \\
\text { Betrieben bei der Zusammenar- } \\
\text { beit? }\end{array}$ \\
\hline & & $\begin{array}{l}\text { Was wünscht sich die GKV von } \\
\text { den Betrieben in der Zusam- } \\
\text { menarbeit? }\end{array}$ \\
\hline \multirow[t]{3}{*}{$\begin{array}{l}\text { Das neue } \\
\text { Präventionsgesetz }\end{array}$} & $\begin{array}{l}\text { Haben Sie im Zusammenhang mit } \\
\text { Prävention schon mal etwas vom } \\
\text { neuen Präventionsgesetz gehört? }\end{array}$ & \multirow{2}{*}{$\begin{array}{l}\text { Im letzten Jahr wurde das neue } \\
\text { Präventionsgesetz } \\
\text { verabschiedet. Hat das Ihren } \\
\text { Arbeitsalltag schon verändert? }\end{array}$} \\
\hline & \multirow{2}{*}{$\begin{array}{l}\text { Falls ja, hat das Gesetz Ihren Alltag } \\
\text { bereits beeinflusst? }\end{array}$} & \\
\hline & & $\begin{array}{l}\text { Was halten Sie von dem Ansatz } \\
\text { eines trägerübergreifenden } \\
\text { Konzepts? }\end{array}$ \\
\hline
\end{tabular}

operationen. Insgesamt wurden 12 Personen aus Nordrhein-Westfalen und Niedersachsen interviewt, darunter 6 Frauen und 6 Männer im Alter von $49( \pm 15)$ Jahren. Die beiden Gruppen (Arbeitgeber, GKV-Vertreter) wurden von je 6 Personen repräsentiert, wobei Vertreter aus verschiedenen Positionen und Abteilungen ausgewählt wurden (s. • Tab. 1). Geschäftsführer, Personalleiter und BGMBeauftragte vertraten die Perspektiven der Arbeitgeber.

Die Kontaktaufnahme und die Terminabsprache erfolgten persönlich, telefonisch oder per E-Mail. Voraussetzung für die Durchführung des Interviews war die Unterzeichnung der Einverständnisund Datenschutzerklärung.

Die Erfassung der Erwartungen fand in Form eines qualitativen, teilstrukturierten Interviews statt. Ein Leitfaden mit offenen Fragen diente als Strukturierungshilfe bei den Interviews und ermöglichte es flexibel an den Forschungsgegenstand heranzugehen und dennoch auf die für die Hauptfragestellung relevanten Themen zu lenken. Für die Befragungsgruppen wurde der Interviewleitfaden jeweils leicht modifiziert.
Der Interviewleitfaden gliederte sich in vier Phasen nach Helfferich [5]. Phase eins diente der Vorstellung des Interviewers und der Erinnerung an das Einholen der formalen Aspekte, der Einverständniserklärung zur Aufzeichnung des Interviews und zum Datenschutz. Neben allgemeinen Angaben zur Person, wie Alter und Geschlecht wurde die Betriebszugehörigkeit erfasst, da sich diese auf Erwartungshaltungen auswirken kann. Eine einfache, spontan ausgerichtete Aufwärm- bzw. Eisbrecherfrage leitete den Einstieg in das eigentliche Interview ein. Die dritte Phase der Erarbeitung beinhaltete die Hauptfragen zu den einzelnen Themenschwerpunkten, welche sich in drei Abschnitte gliederten. Der erste Abschnitt „Prävention im Allgemeinen (1)“ erfragte allgemeine Erwartungen bezüglich der Präventionsmaßnahmen. Der Abschnitt „Präventionsmaßnahmen im Betrieb (2)“ bezog sich speziell auf Erwartungen im Setting Betrieb. Anhand des dritten Abschnitts „das neue Präventionsgesetz (3)“ sollte ein Eindruck zu dem neuen Präventionsgesetz gewonnen werden. Anschließend wurden Erwartungen an bestehen- de Präventionsmaßnahmen sowie bezüglich der Zusammenarbeit mit den anderen Stakeholdern erfragt. In Phase vier wurde den Interviewpartnern die Möglichkeit zu weiteren Ergänzungen gegeben. - Tab. 2 zeigt die Hauptfragen für die Befragungsgruppe der AG und Vertreter der GKV.

Als Ort wurden die Räumlichkeiten der Interviewpartner genutzt. In der Regel gelang es die Interviews ohne große Störungen oder Unterbrechungen innerhalb von 8-25 ( $\varnothing 15)$ min durchzuführen. Der große Unterschied in der Interviewdauer ergab sich aufgrund der offenen und möglichst natürlichen Gesprächsführung.

Bei der Interviewdurchführung wurden die Regeln von Gläser und Laudel [4] berücksichtigt und eine möglichst angenehme und natürliche Gesprächsatmosphäre erzeugt. Zur Vermeidung eines Informationsverlusts wurden alle Interviews aufgezeichnet. Die Interviews wurden nach den Regeln der einfachen Transkription [7] mithilfe der Software easytranscript umgesetzt. Die qualitative Auswertung auf der Grundlage der inhaltlich strukturierten qualitativen Inhaltsanalyse fand mithilfe des Analyseprogramms MAXQDA 12 (VERBI Software GmbH Berlin) statt. Die Bildung und Zuordnung von Kategorien und Subkategorien erfolgte zunächst deduktiv entsprechend der Fragestellung für die Hauptkategorien und wurde auf der Grundlage des Materials induktiv ergänzt. Im Rahmen der Auswertung wurde durch den Austausch im Forschungsprozess und die gegenseitige Kontrolle der Interviewerinnen bei den Analyseschritten die Güte der Daten gewährleistet.

\section{Ergebnisse}

Im Folgenden werden die Ergebnisse bezüglich der Erwartungen an die Zusammenarbeit zur Umsetzung von Präventionsmaßnahmen im betrieblichen Setting aus den beiden Perspektiven der Arbeitgeber und der Vertreter der GKV dargestellt.

Fünf der sechs Arbeitgeber (AG) führten bereits Maßnahmen im Bereich der Prävention und Gesundheitsförderung in ihrem Betrieb durch. In 4 Fällen 
Präv Gesundheitsf 2019 • 14:218-222 https://doi.org/10.1007/s11553-018-0699-1

(c) Der/die Autor(en) 2019

B. Biallas · L. A. L. Dejonghe · J. Meyer · S. Humm · I. Froböse · A. Schaller

\section{Zusammenarbeit in der betrieblichen Gesundheitsförderung - eine qualitative Analyse der Erwartungen von Arbeitgebern und gesetzlichen Krankenkassen}

\section{Zusammenfassung}

Hintergrund. Technologischer Fortschritt, zunehmende Flexibilisierung und erhöhter Wettbewerbsdruck führen zu immer höheren Anforderungen am Arbeitsplatz. Aus diesem Grund wird die Bedeutung der betrieblichen Gesundheitsförderung (BGF) immer wichtiger. Bei der Umsetzung dessen treffen unterschiedliche Interessensgruppen aufeinander. Eine gute Zusammenarbeit ist maßgeblich für eine erfolgreiche Umsetzung von Maßnahmen zur Gesundheitsförderung im Betrieb.

Fragestellung. Welche Erwartungen haben die Arbeitgeber und die Vertreter der gesetzlichen Krankenversicherung (GKV) im Hinblick auf die Zusammenarbeit bei Maßnahmen der BGF?

\begin{abstract}
Methoden. Insgesamt wurden 12 Personen aus Nordrhein-Westfalen und Niedersachsen, darunter 6 Frauen und 6 Männer im Alter von 49 ( \pm 15 ) Jahren interviewt. Die beiden Interessensgruppen wurden durch je 6 Personen repräsentiert.

Ergebnisse. Innerhalb der Interviews zu den Erwartungen bei der Zusammenarbeit zeigen sich sehr konkrete Erwartungen der gesetzlichen Krankenkassenvertreter wie Unterstützung durch die Führungsebene oder eine nachhaltige Verankerung des Themas im Betrieb. Die Arbeitgeber formulieren insbesondere die Erwartungen an eine finanzielle Unterstützung und Beratung bei der Planung und Umsetzung von Maßnahmen.
\end{abstract}

Diskussion. Das Zusammentreffen unterschiedlicher Akteure im Rahmen der BGF kann die Umsetzung von Prozessen und Maßnahmen positiv oder negativ beeinflussen. Somit müssen die Erwartungen aneinander für die Umsetzung einer optimalen Zusammenarbeit geklärt sein. Weitere Kostenträger sind kaum als Partner für die Umsetzung bekannt. Es fehlt an Transparenz und Aufklärung hinsichtlich der Möglichkeiten zur Unterstützung für das Unternehmen.

Schlüsselwörter

Betriebliche Gesundheitsförderung . Interessensgruppen · Trägerübergreifende Zusammenarbeit · Präventionsgesetz . Sozialversicherungsträger

\section{Cooperation in workplace health promotion-a qualitative analysis of the expectations of employers and statutory health insurance companies}

\section{Abstract}

Background. Due to technological progress, increasing flexibility and increased competitive pressure the importance of workplace health promotion is increasingly important. Thus, for optimal workplace health promotion the mutual interaction of different interest groups is of utmost importance.

Objectives. What expectations do the different interest groups (employers and statutory health insurance companies) have with regard to workplace health promotion measures?

Materials and methods. A total of 12 persons from North Rhine-Westphalia and Lower Saxony were interviewed, including 6 women and 6 men aged $49( \pm 15)$ years. The two interest groups were each represented by 6 persons.

Results. The interviews revealed very concrete expectations of the statutory health insurance companies, such as support from the management level or a sustainable anchoring of the topic in the company. In particular, employers formulate their expectations for financial support and advice in the planning and implementation of measures.

Discussion. The meeting of different stakeholders within the framework of workplace health promotion can have a positive or negative influence on the implementation of processes and measures. Thus, the heterogeneous expectations must be clarified for the implementation of the cooperation. Other cost bearers are hardly known as partners for implementation. There is a lack of transparency and clarification regarding the possibilities of support for the company.

Keywords Workplace health promotion - Interest groups . Cross-organizational cooperation · Prevention act . Social insurance carriers war die GKV als Partner beteiligt. Die meisten Teilnehmer nannten zunächst Erwartungen an die Zusammenarbeit mit den GKVen und äußerten sich erst auf Nachfrage auch in Bezug auf andere Sozialversicherungsträger. Insgesamt wurden in der Analyse sechs Subkategorien gebildet (finanzielle Unterstützung, Unterstützung bei der Durchführung/ Maßnahmenangebote, keine Erwartungen [RV und UV], Beratung [GKV und UV], medizinische Ausstattung [RV], Aufklärung über Aufgaben der einzel- nen Sozialversicherungsträger). Bei 4 von 6 Teilnehmern wird die Erwartung „Finanzielle Unterstützung “ besonders häufig genannt. Außerdem erwarten 3 der befragten Personen auch eine „Unterstützung bei der Durchführung/ Maßnahmenangebote“.

„Wenn ich jetzt mit einem meiner Ansprechpartner in Kontakt bin erwarte ich natürlich schon, wenn ich das Thema Rücken einwerfe (...), dass er dann auch mit einigen Vorschlägen kommt. " $(A G)$
In Bezug auf die Unfall- und Rentenversicherung zeigt sich, dass die Interviewteilnehmer "keine speziellen Erwartungen“ an diese Träger äußern.

„Also jetzt im Moment würden Sie noch nicht wirklich Unterstützung von denjenigen erwarten. " $(A G)$

Nur einzelne Interviewteilnehmer erwarten eine „Beratung“ von gesetzlichen Unfallversicherungen und Krankenkassen, sowie eine Unterstützung der gesetzlichen Rentenversicherung im Hinblick 
auf medizinische Ausstattung. Mehrere Arbeitgeber äußern hingegen den Wunsch einer besseren „Aufklärung über Aufgaben der einzelnen Sozialversicherungsträger“.

„Also was ich gerne ergänzen würde, ist ganz einfach, dass hinsichtlich des Präventionsgesetztes noch sehr viel Aufklärungsbedarf ist (...). Da fehlt einfach irgendwie noch so eine Art Handlungsleitfaden, (...) wodurch auch wirklich jeder Kleinunternehmer weiß, das steht mir zu, das muss ich machen, damit ich das hinbekomme und hier kriege ich die und die Hilfe." (AG)

Im Hinblick auf die trägerübergreifende Zusammenarbeit erwartet ein Arbeitgeber „Schwierigkeiten bei der Vernetzung der Träger" und betont, viel Wert auf eine gute Qualität bei der Zusammenarbeit zu legen:

„Es wird dadurch ja nicht erstmal per se besser, dass man sagt, so ich habe jetzt andere Leute. Das wird eher die Schwierigkeit dann sein, dass mal wieder zu vernetzen und zu gucken." (AG)

Die Erwartungen der Vertreter der GKV an die Zusammenarbeit mit den Betrieben lassen sich in sechs Subkategorien abbilden (Ressourcenbeteiligung/ eigenes Engagement, Unterstützung Führungsebene, Nachhaltigkeit/strukturelle Verankerung, Ansprechpartner im Betrieb, zur Krankenkasse als Partner stehen, Kontakt zu den Mitarbeitern). Am häufigsten wird eine „Ressourcenbeteiligung/eigenes Engagement" seitens der Betriebe erwartet. Drei Interviewteilnehmer nennen außerdem die „Unterstützung der Führungsebene":

„Also wir erwarten, dass wir das nicht komplett selbst organisieren müssen, sondern, dass unterstützt wird, vor allem von oben." (GKV)

Ebenfalls drei der Krankenkassenvertreter beziehen sich auf eine „Nachhaltigkeit/strukturelle Verbesserung " der Maßnahmen in den Unternehmen und erwähnen dabei unter anderem die Notwendigkeit von „Ansprechpartnern vor Ort".
„Ich erwarte (...), dass es jemanden gibt, der sich verantwortlich fühlt, mit dem wir das besprechen können, der auch die Kompetenz übertragen bekommt von dem Unternehmen und so weiter." (GKV)

Zwei Interviewteilnehmer nennen darüber hinaus den Wunsch „zur Krankenkasse als Partner stehen“.

„Natürlich erwarten wir, dass die Betriebe das auch kommunizieren mögen, dass wir diese Maßnahmen unterstützen, das ist uns natürlich ganz wichtig. (...) Wir erwarten tatsächlich auch, dass das Unternehmen sich zu uns bekennt, als Partner in Sachen Gesundheit" (GKV)

Lediglich ein Teilnehmer äußert die Erwartung „Kontakt zu den Mitarbeitern“ und erhofft sich eine direkte freie Kommunikation mit den Beschäftigten.

Bezogen auf „Erwartungen an die trägerübergreifende Zusammenarbeit“ gehen jeweils vier Vertreter hierbei sowohl von Vorteilen durch eine „Ergänzung der Träger“, als auch „Schwierigkeiten“ aus. Dies bildet sich in „Zunahme der trägerübergreifenden Projektförderung“ sowie in „besseres Erreichen vulnerabler Gruppen“ ab. Als Befürchtungen werden „Berührungsängste“ genannt. Diese bestehen aufgrund finanzieller Ressourcen ebenso wie aufgrund einer fehlenden Abstimmung. Ein Vertreter macht deutlich sich in der alltäglichen Arbeit noch nicht mit diesem Ansatz beschäftig zu haben, sodass er „keine Erwartungen“ hat.

\section{Diskussion}

Innerhalb der Interviews zu den Erwartungen bei der Zusammenarbeit der Stakeholder im Bereich der BGF zeigen sich sehr konkrete Erwartungen und Wünsche der GKV-Vertreter, während die Arbeitgeber sich eher allgemein auf die gewünschten Leistungen beziehen oder keine präzisen Erwartungen formulieren. Die beidseitige Betonung der finanziellen Unterstützung macht deutlich, dass die Bereitschaft, finanzielle Ressourcen zur Verfügung zu stellen, bei den interviewten Arbeitgebern scheinbar nur teilweise vorhanden ist. Zudem zeigte sich bei den Arbeitgebern noch
Informationsbedarf in Bezug auf Leistungen und Unterstützungsmöglichkeiten der einzelnen Sozialversicherungsträger, insbesondere $\mathrm{zu}$ den Leistungen der gesetzlichen Unfall- und Rentenversicherungen. Es bedarf weiterhin an stetiger Aufklärungsarbeit [1]. Dabei ist $\mathrm{zu}$ vermuten, dass nicht nur die Zusammenarbeit zwischen Unternehmen und Sozialversicherungsträgern aufeinander abgestimmt werden muss, sondern auch die Zusammenarbeit der einzelnen Sozialversicherungsträger, die durch das Präventionsgesetz [12] manifestiert ist. Auch die Zusammenarbeit der GKVen miteinander sollte optimiert werden, um den Missbrauch der BGF im Wettbewerb zwischen den GKVen als Marketinginstrumente entgegenzuwirken [6]. Zudem stellt eine Standardisierung der Maßnahmenqualität eine Möglichkeit für trägerübergreifende Akzeptanz der Ansätze anderer Akteure im Feld dar [11]. Die Errichtung der Koordinierungsstellen sowie die gemeinsame Erstellung der Landesrahmenempfehlungen zeigen die ersten Schritte hinsichtlich einer aktiven Zusammenarbeit der Kostenträger. Es wird Zeit benötigen bis sich Prozesse und Abläufe eingespielt haben [9]. Ziel sollte es sein, die Prozesse zwischen den Kostenträgern und entsprechend die trägerübergreifende Zusammenarbeit für die Unternehmen transparenter $\mathrm{zu}$ gestalten. Inwiefern sich eine $\mathrm{Zu}$ sammenarbeit auch mit Partnern wie örtliche Unternehmensorganisationen, Industrie- und Handelskammern sowie Handwerkskammern und Innungen vor Ort vermehrt wiederfindet, wird sich zeigen. Die vorliegenden Interviews machen deutlich, dass diese Kooperationspartner bisher scheinbar wenig Beachtung finden.

Die Ergebnisse wurden durch qualitative Interviews mit einer recht kleinen Stichprobe ermittelt. Um die Ergebnisse zu bestätigen, sollten weitere Untersuchungen erfolgen. In Bezug auf die Stichprobe ist anzumerken, dass die Interviewteilnehmer aufgrund bestehender Kontakte akquiriert wurden. Es ist daher nicht auszuschließen, dass diese bereits ein höheres Interesse an der Thematik besessen haben. Außerdem zeigt sich ein großer Unterschied in der Dauer der Betriebszu- 
gehörigkeit (1-40 Jahre). Dies kann sich auf persönliche Erwartungen auswirken und sollte in zukünftigen Studien beachtet werden. Ein weiterer zu berücksichtigender Faktor ist die Alterspanne der Interviewten (31-62 Jahre). Auch das Alter kann Erwartungshaltungen beeinflussen. Weitere Arbeiten sollten dies verstärkt berücksichtigen. Zudem sollten neben den GKVen weitere Sozialversicherungsträger befragt werden, um die trägerübergreifende Zusammenarbeit zu analysieren. Aufgrund der bisher unzureichenden Studienlage sind weitere Untersuchungen zu dieser Thematik notwendig. Dabei könnte es von Vorteil sein, eine Differenzierung der Arbeitgeber nach Branchen und Betriebsgrößen vorzunehmen, um spezifischere Aussagen zu Erwartungen treffen zu können. Interessant wäre, inwiefern sich die Stakeholder über gegenseitige Erwartungen bewusst sind und welche konkreten Förder- und Hemmfaktoren in der Zusammenarbeit bestehen. So können Lösungen gefunden und die Zusammenarbeit optimiert werden. Insgesamt zeigt sich die Klärung der Erwartungshaltung als wichtige Grundvoraussetzung im Rahmen der Zusammenarbeit verschiedener Stakeholder zur Implementierung und Umsetzung von BGF. Nur durch eine optimale Zusammenarbeit steigt die Qualität der BGF im Sinne des Arbeitnehmers und einer nachhaltigen strukturellen Etablierung des Gesundheitsthemas im betrieblichen Setting.

\section{Fazit für die Praxis}

- Es zeigt sich, dass die Erwartungen an eine Zusammenarbeit sich zwischen Arbeitgebern und GKVen unterscheiden.

- Ein vermehrter Austausch von Erwartungen und Wünschen unterschiedlicher Interessensgruppen scheint notwendig, um die Umsetzung von Maßnahmen der BGF zu optimieren.

- Eine Aufklärung der Arbeitnehmer über die möglichen Leistungen und Beiträge unterschiedlicher Kostenträger sollte erfolgen.

- Die Zusammenarbeit zwischen den GKVen untereinander und mit den weiteren Kostenträgern sollte im
Sinne des Arbeitgebers und auch des Arbeitnehmers weiter forciert werden.

\begin{tabular}{ll}
\hline Korrespondenzadresse \\
\hline $\begin{array}{l}\text { Dr. Bianca Biallas } \\
\text { Institut für Bewegungsthe- } \\
\text { rapie und bewegungsori- } \\
\text { entierte Prävention und } \\
\text { Rehabilitation, Deutsche } \\
\text { Sporthochschule Köln } \\
\text { Köln, Deutschland } \\
\text { biallas@dshs-koeln.de }\end{array}$ \\
\hline
\end{tabular}

Förderung. BMBF Fördernummer 01EL1425A

\section{Einhaltung ethischer Richtlinien}

Interessenkonflikt. B. Biallas, L.A.L. Dejonghe, J. Meyer, S. Humm, I. Froböse und A. Schaller geben an, dass kein Interessenkonflikt besteht.

Dieser Beitrag beinhaltet keine von den Autoren durchgeführten Studien an Menschen oder Tieren.

Open Access Dieser Artikel wird unter der Creative Commons Namensnennung 4.0 International Lizenz (http://creativecommons.org/licenses/by/4.0/deed. de) veröffentlicht, welche die Nutzung, Vervielfältigung, Bearbeitung, Verbreitung und Wiedergabe in jeglichem Medium und Format erlaubt, sofern Sie den/die ursprünglichen Autor(en) und die Quelle ordnungsgemäßnennen, einen Linkzur Creative Commons Lizenz beifügen und angeben, ob Änderungen vorgenommen wurden.

\section{Literatur}

1. Bechmann $S$, Jäckle $R$, Lück $P$, Herdegen $R$ (2001) Motive und Hemmnisse für Betriebliches Gesundheitsmanagement (BGM). Umfrage und Empfehlungen, 2. Aufl. iga.Report, Bd. 20. AOKBundesverband, BKK Bundesverband, Deutsche Gesetzliche Unfallversicherung (DGUV) und Verband der Ersatzkassen e.V. (vdek) (Hrsg), Berlin

2. Bräunig D, Haupt J, Kohstall T et al (2015) Wirksamkeit und Nutzen betrieblicher Prävention. iga.Report, Bd. 28, AOK-Bundesverband, BKK Bundesverband, Deutsche Gesetzliche Unfallversicherung (DGUV) und Verband der Ersatzkassen e.V. (vdek) (Hrsg), Berlin

3. Faller G, Abel B, Badura B et al (Hrsg) (2016) Lehrbuch betriebliche Gesundheitsförderung, 3. Aufl. Hogrefe, Bern

4. Gläser JLG (2010) Experteninterviews und qualitative Inhaltsanalyse als Instrumente rekonstruierender Untersuchungen, 4. Aufl. VS, Wiesbaden (Lehrbuch)

5. Helfferich C (2011) Die Qualität qualitativer Daten. VS, Wiesbaden

6. Jena M, Di Pasquale V (2014) Betriebliche Gesundheitsförderung - die Perspektive der Gewerkschaften. In: Hahnzog S (Hrsg) Betriebliche Gesundheitsförderung. Das Praxishandbuch für den Mittelstand. Springer Gabler, Wiesbaden, S37-47

7. Kuckartz U (2007) Qualitative Evaluation. Der Einstieg in die Praxis. VS; GWV Fachverlage GmbH, Wiesbaden

8. Lenhardt U (2012) Akteuere der Betrieblichen Gesundheitsförderung: Interessenlagen - Handlungsbedingungen - Sichtweisen. In: Faller G (Hrsg) Lehrbuch betriebliche Gesundheitsförderung, 2. Aufl. Huber, Bern

9. Medizinischer Dienst des Spitzenverbandes Bund der Krankenkassen e. V. (2016) Präventionsbericht 2016 - Leistungen der gesetzlichen Krankenversicherung: Primärprävention und betriebliche Gesundheitsförderung Berichtsjahr 2015. Berlin, Essen

10. Nationale Präventionskonferenz (2016) Bundesrahmenempfehlungen der Nationalen Präventionskonferenz nach $\S 20$ d Abs. 3 SGB V. Berlin

11. Noweski M, Bär G, Voss A, Ihm M, Fricke L (2018) Qualitätsstandards in der Primärprävention. Prävention Gesundheitsförderung 13(2):97-102

12. PrävG (2015) Gesetzzur Stärkung derGesundheitsförderung und der Prävention (Präventionsgesetz). Bundesgesetzblatt, Teil 1

13. Schaefer E, Drexler H, Kiesel J (2016) Betriebliche Gesundheitsförderung in kleinen, mittleren und großen Unternehmen des Gesundheitssektors Häufigkeit, Handlungsgründe der Unternehmensleitungen und Hürden der Realisierung (Workplace Health Promotion in Small, Medium-Sized and Large Enterprises of the Health-Care Sector - Frequency, Reasons for the Company Management to Take Action and Barriers to Implementation). Gesundheitswesen 78(3):161-165. https://doi. org/10.1055/s-0034-1396887

14. Uhle TTM (2011) Betriebliches Gesundheitsmanagement. Springer, Berlin, Heidelberg

15. Zok K (2009) Stellenwert und Nutzen betrieblicher Gesundheitsförderung aus Sicht der Arbeitnehmer. In: Badura B, Schröder H, Vetter C (Hrsg) Betriebliches Gesundheitsmanagement: Kosten und Nutzen. Springer, Berlin, Heidelberg 